\title{
Análisis del consumo de drogas legales como el alcohol, el ta- baco y los psicofármacos, y la percepción del riesgo en jóvenes universitarios
}

\author{
Rosario Ruiz-Olivares $^{1^{*}}$, Valentina Lucena ${ }^{2}$, M. José Pino ${ }^{1}$, and Javier Herruzo ${ }^{1}$ \\ ${ }^{1}$ Universidad de Córdoba ${ }^{2}$ Centro Renacer (Córdoba) \\ (Received January 13, 2010; Accepted March 8, 2010)
}

RESUMEN: El objetivo de este trabajo es relacionar el consumo de sustancias como el alcohol, el tabaco, y los psicofármacos con la percepción de riesgos como, sufrir un accidente de tráfico, padecer problemas de salud transitorios, problemas legales, dificultad en las relaciones interpersonales, disminución de la capacidad para realizar tareas y problemas de salud graves e irreversibles. Con un diseño expost-facto prospectivo de grupo único (Montero y León, 2007), y un cuestionario elaborado a partir del cuestionario utilizado en el estudio "Andaluces ante las drogas" (Navarro, Alonso y Fernández, 2005), se recogió información sociodemográfica y sobre la percepción de riesgo ante el consumo de distintas sustancias de 1011 estudiantes de la Universidad de Córdoba. Los resultados señalan que en general los jóvenes universitarios que más consumen alcohol, tabaco y psicofármacos son los que valoran con una puntuación más baja los posibles riesgos derivados del consumo. Además, el modelo de regresión para cada una de las sustancias indica que una valoración baja en determinados riesgos puede predecir un mayor consumo de la misma. En conclusión, dadas las tendencias de consumo de drogas en estudiantes universitarios y la baja percepción del riesgo de ciertas consecuencias negativas derivadas del mismo sería conveniente poner en marcha estrategias preventivas en el ámbito universitario.

Palabras clave: percepción del riesgo, consumo de alcohol, tabaco y psicofármacos, universitarios.

Analysis of legal drugs consumption like alcohol, tobacco and psycho-drugs, and risk perception in University young people

ABSTRACT: The aim of this study is to relate the consumption of legal substances like alcohol, cigarettes, and psycho-drugs with the perception of risks such like traffic accidents, the suffering of temporary health problems, legal problems, difficulty in the interpersonal relations, decrease of the capacity to realize tasks and irreversible health problems. With a prospective expost-facto design of only one group (Montero \& León, 2007), and a questionnaire elaborated from the questionnaire used in the study "Andaluces ante las drogas" (2005), relevant socio-demographic and risk perception information was collected regarding the consumption of different substances in 1011 students of the University of Córdoba. The results revealed university people that generally consume more alcohol, cigarettes and psycho-drugs are those that value with a lower punctuation the possible risks derived from the consumption. Besides, the model of regression for each one of the substances indicated that a low assessment in determined 
risks can predict a main consumption of these. As a conclusion, given the trends of drugs consumption in university students and the low perception of the risk of its negative consequences, it would be convenient to set up preventive strategies in the university field.

Key words: risk perception, alcohol consumption, cigarettes and psyco-drugs, University students.

\section{INTRODUCCIÓN}

El consumo de drogas en jóvenes y adolescentes se vincula, hoy en día, a un estilo de ocio recreativo compartido con el grupo de iguales, con búsqueda de la sobre-estimulación y como mecanismo de reafirmación de la identidad grupal (Geoffrey, Kristin y Faith, 2008; Moral, Rodríguez y Sirvent, 2006). Es lícito pensar que las personas toman decisiones en función de las consecuencias positivas o negativas que éstas les van a proporcionar, evitando aquellos comportamientos que les aporten consecuencias negativas (Becoña, 2000). Una de la variables que interviene en esa toma de decisiones para el consumo de sustancias es la percepción del riesgo, entendida como el grado en que se atribuye a una conducta un supuesto peligro para la salud (Becoña, 2000). Es bien sabido que el consumo de sustancias tiene efectos sobre la salud de forma directa (sobredosis, enfermedades cardiovasculares, cáncer, etc.) e indirecta (accidentes de tráfico, deterioro en las relaciones interpersonales, bajo rendimiento escolar, etc.) (Ballester, Gil y Guirado, 2000). Sin embargo, los jóvenes tienden a experimentar con este tipo de actividades de alto riesgo a pesar de conocer algunas de sus posibles consecuencias. Parker, Aldridge, y Measham (1998) sugieren que los jóvenes tienden a experimentar con estas actividades de alto riesgo, pues el consumo no se halla tanto ligado a una respuesta pasiva frente a la oportunidad y/o incentivo de su uso, sino que las decisiones sobre el uso de drogas están relacionadas con las apreciaciones de beneficio y riesgo que el consumo supone. Hay adolescentes que rechazan ciertas drogas, a pesar de las oportunidades que tienen para utilizarlas (Megías, 2004). En un estudio realizado con estudiantes de secundaría de Andalucía sobre el fenómeno del botellón, como factor de protección entre otros está la percepción de que el consumo de alcohol al participar en el botellón conlleva numerosos efectos negativos (Gil-Flores, 2008).

Las mayores diferencias en la percepción del riesgo asociado al consumo de drogas no se establecen con el patrón de consumo, sino en relación con el tipo de droga consumida (Fernández et al., 2003). La concepción que se tiene sobre las diferentes drogas, que a su vez dependerá tanto del uso, como de las creencias y de la propia construcción social de la sustancia, será determinante en el consumo de las mismas (Becoña, 2000). Según la Encuesta Estatal sobre Uso de Drogas en Enseñanzas Secundarias (Ministerio de Sanidad y Consumo, 2005) en España, el consumo de drogas de comercio legal (alcohol, tabaco y psicofármacos) se asocia con un menor riesgo que el consumo de drogas de comercio ilegal (Álvarez, Fraile, Secades, Vallejo y Fernández, 2005). En función del sexo, parece que las 
mujeres perciben un mayor riesgo para todas las sustancias, excepto en el caso de los psicofármacos donde las diferencias fueron inapreciables. Además, conforme aumenta la edad del encuestado disminuye el riesgo percibido ante el consumo drogas (Ministerio de Sanidad y Consumo, 2005) y aumenta el consumo de las mismas, produciéndose lo que algunos autores señalan como banalización del consumo (Ministerio de Sanidad y Consumo, 2005). Según el informe de la encuesta domiciliaria sobre Alcohol y Drogas en España (Ministerio de Sanidad y Consumo, 2007) a adolescentes de 14 y 18 años, las conductas de consumo consideradas más peligrosas son el consumo habitual de sustancias ilegales como la heroína o los alucinógenos, y las menos peligrosas el consumo de cinco o seis cañas o copas en un fin de semana, y el consumo esporádico de psicofármacos o cannabis. La percepción del riesgo de consumir un paquete de tabaco al día se ha visto incrementada.

Algunos trabajos científicos hacen referencia a la relación entre una baja percepción del riesgo y el consumo de sustancias en población adolescente (Álvarez, Fraile, Secades, Vallejo y Fernández, 2005; Espada, Pereira y GarcíaFernández, 2008; Geoffrey et al., 2008; Hernández y Solano, 2007; Martins, Store, Alexandre y Chilcoat, 2008; Moral, Ovejero y Pastor, 2004; Moral et al., 2006, Rodríguez et al., 2008; Trujillo, Forns y Pérez, 2007). En un estudio realizado con adolescentes sobre actitudes y consumo de sustancias psicoactivas por niveles de edad, se confirma que la percepción de riesgo distorsionada sobre las sustancias (reestructuraciones cognitivas, ilusiones de invulnerabilidad) es el principal factor de inicio de experimentación en el consumo de alcohol y tabaco en los más jóvenes (Moral y Ovejero, 2005). Así, el riesgo percibido ante distintas conductas de consumo de drogas puede ser un indicador indirecto de la evolución presente o futura de la prevalencia de consumo en jóvenes. Con el alcohol parece que los jóvenes que mayor consumo presentan suelen tener una menor percepción de los riesgos. En el trabajo de Espada et al. (2008) se observó cómo 536 estudiantes de ESO no percibían el alcohol como una sustancia nociva, sólo se consideraba peligroso cuando el padre sí era consumidor, y menos peligroso cuando el consumidor era su mejor amigo. Otro trabajo con adolescentes de 12 a 19 años (Moral et al., 2006), ha explorado la percepción de riesgos y la conciencia de daño físico y/o psicosocial derivado de la experimentación con alcohol, entre consumidores de alcohol y de alcohol/cocaína. Se confirma que una actitud de mayor permisividad y percepción de riesgos distorsionada se asocia a un mayor consumo de alcohol y alcohol/cocaína.

En definitiva, aquellos individuos que abusan de las drogas tienen unas expectativas diferentes de los no consumidores respecto a los probables efectos derivados de su empleo (Novacek, Raskin y Hogan, 1991). Mientras éstos muestran actitudes de resistencia a la experimentación, los consumidores reelaboran las percepciones de riesgo y valoran las consecuencias positivas del consumo de drogas (Moral et al., 2006). Según un estudio realizado entre consumo de esteroides y percepción de riesgo de Irigoyen-Coll (2005), se observó que a medida que bajaba la percepción del riesgo sobre los efectos negativos en la salud 
aumentaba el consumo. Así, del 5,2\% de los consumidores, el 4,1 \% poseía baja percepción del riesgo y entre los no consumidores, un 20,3\% presentaba baja percepción del riesgo asociado a las consecuencias negativas en la salud, encontrándose en condición de vulnerabilidad.

Hay estudios realizados con población universitaria, donde se confirman patrones de consumo de cierta importancia, sobre todo en lo que se refiere a drogas legales (Jiménez-Muro, Belmonte, Marqueta, Gargallo y Nerín, 2009). El riesgo percibido ante distintas conductas de consumo de drogas puede ser un indicador indirecto de la evolución del consumo. Así, el consumo de drogas legales como el alcohol, el tabaco y los psicofármacos, podría facilitar el posterior consumo de cannabis, considerada primera droga ilegal consumida en España. En el trabajo de Jiménez-Muro et al. (2009) con alumnos que ingresaban por primera vez en la Universidad de Zaragoza, se observó cómo los jóvenes, en cuanto a la percepción del riesgo, consideraban que la enfermedad y la muerte eran fenómenos muy lejanos en sus vidas por lo que no valoraban los riesgos que conllevaba el consumo de sustancias como el alcohol y el tabaco.

Por todo esto, conocer qué factores están asociados al consumo de sustancias de carácter legal como el alcohol, el tabaco y los psicofármacos en jóvenes universitarios puede contribuir a mejorar las actuaciones preventivas dirigidas a este colectivo de jóvenes. A pesar de que un nivel educativo alto funciona como factor protector del consumo de sustancias (García, Guerra-Gutiérrez, OrtegaMartínez, Sánchez-Villegas y Martínez-González, 2004; Guillén, Nerín, Mas y Crucelaegui, 2003; Mas et al., 2004), un gran número de jóvenes que accede a estudios superiores inicia y/o consolida una conducta de consumo a lo largo de su etapa universitaria (Cunill, Planes y Grass, 1998; Font-Mayolas, Grass y Planes, 2006; Jiménez-Muro et al., 2009).

El objetivo de este trabajo de investigación es relacionar el consumo de sustancias de jóvenes universitarios de la Universidad de Córdoba como el alcohol, el tabaco y los psicofármacos con la percepción de riesgos directos e indirectos de su consumo como, sufrir un accidente de tráfico, padecer problemas de salud transitorios, problemas legales, dificultad en las relaciones interpersonales, disminución en la capacidad para realizar tareas y problemas de salud graves e irreversibles.

\section{MÉTODO}

\section{Diseño}

El estudio se ha realizado mediante un diseño expost-facto prospectivo de grupo único (Montero y León, 2007). La población objetivo eran 15.000 alumnos de la Universidad de Córdoba (UCO). El proyecto se planteó con el visto bueno del Vicerrectorado de Estudiantes de la UCO que solicitó mediante un escrito formal la colaboración de los decanos y administradores de todos los centros. Para la predeterminación del tamaño de la muestra, se partió de la siguiente simulación realizada con el paquete estadístico EpiInfo 2000, que para un 1\% de 
frecuencia esperada y un margen de error del 0,01, se obtuvo que como mínimo se debía recoger la información de 1000 sujetos. El muestreo se hizo estratificado por centros, teniendo en cuenta el número de matrículas del curso anterior.

\section{Participantes}

En el estudio han participado 1011 (42\% hombres y 56\% mujeres) estudiantes universitarios de los diferentes centros que componen la UCO, de los cuales el 59,3\% estudian una carrera de las macroáreas de ciencias y el 47\% una carrera de las macroáreas de humanidades, ciencias sociales y jurídicas. Por edades la muestra se distribuye en un 53\% de 18-20 años, un 30,6\% de 21-23 años, un $11,1 \%$ de $24-26$ años, un $5,1 \%>$ de 27 años.

\section{Instrumento y recogida de datos}

Las principales fuentes para elaborar el cuestionario fueron la encuesta sobre drogas del Plan Nacional y del Plan Andaluz (Navarro et al., 2005), y el cuestionario utilizado en el trabajo de Arenas, Carmona, Rodríguez y Tatnell (2002) con población universitaria de la ciudad de Córdoba que tenía como objetivo describir los patrones de consumo de las diferentes sustancias en función de variables como la edad, sexo, estudios universitarios, religión, política, características familiares y nivel socioeconómico dirigido hacia futuras campañas de prevención en el ámbito universitario. El cuestionario final contenía varios bloques de preguntas: a) datos sociodemográficos; b) patrones de consumo, y c) percepción del riesgo de problemas asociados al consumo de sustancias, como accidentes de tráfico, problemas psicológicos o físicos importantes, problemas en las relaciones interpersonales, problemas legales, disminución de ciertas capacidades para realizar una tarea y problemas de salud irreversibles, con una escala del 1 al 5 siendo 1 percepción de bajo riesgo y 5 máxima percepción de riesgo.

La recogida de datos se realizó durante las dos primeras semanas del curso 2007-2008 durante las clases. El profesorado previamente informado, dejó media hora de su clase para que los alumnos contestaran el cuestionario. En el apartado de instrucciones del mismo se especificaba que era una encuesta sobre el consumo de diversas sustancias y algunos comportamientos en población universitaria, cuyo objetivo era la realización de una serie de actuaciones de prevención y tratamiento ante los problemas que pueden derivarse de estas conductas. Por último, se resaltaba la importancia de su participación, informando a su vez que el tratamiento de los datos sería procesado estadísticamente de forma completamente anónima.

Análisis de datos

Con la información proporcionada por los cuestionarios, se elaboró una base 
de datos en SPSS 12.0. Para relacionar el consumo de las sustancias con las distintas variables de percepción de riesgo se utilizó, primero un análisis de la varianza (ANOVA) para observar si se daban diferencias entre los grupos de consumo (nunca, esporádica y habitualmente) y las puntuaciones medias de cada grupo de consumo para cada uno de los riegos. Además, se testaron diversos modelos mediante un análisis de regresión logística para cada una de las variables consumo de alcohol, tabaco, y psicofármacos. Los posibles riesgos asociados fueron tener un accidente de tráfico, problemas legales, problemas de salud transitorios, problemas de relaciones interpersonales, problemas de capacidad para realizar una tarea y problemas de salud irreversibles.

\section{RESULTADOS}

Para valorar la frecuencia con la que consumen los jóvenes universitarios se estableció una escala de respuesta con las siguientes opciones: 1) No, nunca, 2) Sólo lo he probado una vez, 3) Esporádicamente, 4) Sólo los fines de semana, 5) Los fines de semana y algunos días entre semana, 6) Todos los días de la semana. Sin embargo, para calcular el ANOVA, se agruparon los datos en tres categorías: una de no consumo donde se aglutinaban los datos de la respuesta "no, nunca" y "sólo lo he probado una vez", otra que era la opción directa de esporádicamente, y por último, una categoría de consumo habitual donde se unieron las respuestas de las opciones "solo los fines de semana", "los fines de semana y algunos días entre semana" y "todos los días de la semana".

La frecuencia de consumo de sustancias en esta muestra de estudiantes universitarios ha sido, para el tabaco, el $67,2 \%$ no fuman, el 10,3\% lo hacen esporádicamente y un 22,5\% habitualmente. Para el alcohol, el $16,5 \%$ no consume, frente al $28,8 \%$ que lo hace esporádicamente y el $54,7 \%$ que lo hace habitualmente. Por último, el consumo de psicofármacos presenta un $92,4 \%$ de jóvenes que no consumen, un $6,33 \%$ que lo hace esporádicamente y un $1,3 \%$ habitualmente.

Según el ANOVA, cuando se compara el consumo de alcohol (ver tabla1) con los distintos riesgos, no hay diferencias entre los grupos de consumo en cuanto a los problemas de tráfico, pues todos identifican riesgo de tener un accidente de tráfico cuando se consume alcohol $[F(2,992)=2,791 ; p<0,001]$. Sin embargo, cuando los posibles riesgos son problemas psicológicos o físicos importantes $[F(2,987)=12,465 ; p<0,001]$, problemas en las relaciones interpersonales $[F(2,977)=17,257 ; p<0,001]$, problemas legales $[F(2,981)=6,779 ; p<0,001]$, disminución de ciertas capacidades para realizar una tarea $[(\mathrm{F}(2,970)=8,072$; $\mathrm{p}<0,001]$ y problemas de salud irreversibles $[\mathrm{F} 2,950]=18,675 ; \mathrm{p}<0,001]$, el grupo que consume habitualmente los valora con una puntuación baja y aquellos grupos que consumen esporádicamente o no consumen nunca los valora con una puntuación más alta, dichas diferencias son estadísticamente significativas. Atendiendo al análisis de comparaciones múltiples post-hoc (S-N-K) para los 
riesgos, se confirma que no hay diferencias entre el grupo que consume esporádicamente y el que no consume.

Tabla 1. Análisis de Varianza entre el Consumo de Alcohol de Jóvenes Universitarios de la Universidad de Córdoba y la Valoración de Posibles Riesgos Asociados

\begin{tabular}{|c|c|c|c|c|}
\hline RIESGOS & $\begin{array}{l}\text { NIVEL DE } \\
\text { CONSUMO }\end{array}$ & $\begin{array}{l}\text { PUNTUACION } \\
\text { MEDIA (1 a 5) }\end{array}$ & $F(2,998)$ & $\mathbf{P}$ \\
\hline \multirow{3}{*}{ Accidentes de tráfico } & Nunca & 4,78 & \multirow{3}{*}{2,791} & \multirow{3}{*}{0,062} \\
\hline & Esporádicamente & 4,84 & & \\
\hline & Habitualmente & 4,74 & & \\
\hline \multirow{3}{*}{$\begin{array}{l}\text { Problemas psicológicos y } \\
\text { físicos graves }\end{array}$} & Nunca & 4,25 & \multirow{3}{*}{12,465} & \multirow{3}{*}{$0,000^{*}$} \\
\hline & Esporádicamente & 4,25 & & \\
\hline & Habitualmente & 3,93 & & \\
\hline \multirow{3}{*}{$\begin{array}{l}\text { Problemas con las relaciones } \\
\text { interpersonales }\end{array}$} & Nunca & 4,10 & \multirow{3}{*}{17,257} & \multirow{3}{*}{$0,000^{*}$} \\
\hline & Esporádicamente & 3,93 & & \\
\hline & Habitualmente & 3,53 & & \\
\hline \multirow{3}{*}{ Problemas legales } & Nunca & 4,33 & \multirow{3}{*}{6,779} & \multirow{3}{*}{$0,001^{*}$} \\
\hline & Esporádicamente & 4,32 & & \\
\hline & Habitualmente & 4,05 & & \\
\hline \multirow{3}{*}{$\begin{array}{l}\text { Disminución de las } \\
\text { capacidades }\end{array}$} & Nunca & 4,66 & \multirow{3}{*}{8,072} & \multirow{3}{*}{$0,000^{*}$} \\
\hline & Esporádicamente & 4,61 & & \\
\hline & Habitualmente & 4,41 & & \\
\hline \multirow{3}{*}{$\begin{array}{l}\text { Problemas graves para la } \\
\text { salud }\end{array}$} & Nunca & 4,23 & \multirow{3}{*}{18,675} & \multirow{3}{*}{$0,000^{*}$} \\
\hline & Esporádicamente & 4,36 & & \\
\hline & Habitualmente & 3,93 & & \\
\hline
\end{tabular}

$* \mathrm{P}<0,001$

Para el tabaco (ver tabla 2) no hay diferencias significativas entre los tres grupos de consumo para los riesgos asociados a problemas físicos y psíquicos transitorios graves, y problemas de salud graves e irreversibles. Sin embargo, sí hay diferencias entre grupos cuando los riesgos están relacionados con problemas de tráfico $[\mathrm{F}(2,989)=21,930 ; \mathrm{p}<0,001]$, con problemas en las relaciones interpersonales $[\mathrm{F}(2,978)=16,446 ; \mathrm{p}=0,000]$, problemas legales $[\mathrm{F}(2,980)=8,875$; $\mathrm{p}<0,001]$, y problemas relacionados con la capacidad para realizar alguna tarea $[F(2,970)=19.179 ; p<0,001]$. Atendiendo al análisis de comparaciones múltiples post-hoc (S-N-K) para los riesgos, no hay diferencias entre los grupos que consumen esporádica y habitualmente, pues ambos valoran de forma similar con una menor puntuación el consumo de tabaco y el riesgo a tener problemas interpersonales, legales o de capacidad a la hora de desempeñar una tarea.

Para los psicofármacos (véase tabla 3), en general los consumidores de psicofármacos perciben menos riesgos que los no consumidores. Se dan diferencias significativas en todos los riesgos: problemas de tráfico $[\mathrm{F}(2,980)=9,860$; $\mathrm{p}<0,001]$, problemas de salud física y psíquica transitoria $[\mathrm{F}(2,983)=13,511$; $\mathrm{p}<0,001]$, problemas con las relaciones interpersonales $[\mathrm{F}(2,972)=20,357 ; \mathrm{p}=$ $0,000]$, problemas de tipo legal $[\mathrm{F}(2,976)=15,295 ; \mathrm{p}<0,001]$, problemas de capacidad para realizar alguna actividad $[\mathrm{F}(2,971)=24,853 ; \mathrm{p}<0,001]$, riesgo a padecer pérdida de salud irreversible $[\mathrm{F}(2,945)=11,592 ; \mathrm{p}<0,001]$. El análisis de comparaciones múltiples post-hoc $(\mathrm{S}-\mathrm{N}-\mathrm{K})$ confirma que el grupo que mayor 
riesgo percibe es el que no consume, y en algunas ocasiones el que consume esporádicamente. El grupo que consume habitualmente siempre percibe y valora con una puntuación sensiblemente menor los distintos riesgos asociados al consumo de psicofármacos.

Tabla 2. Análisis de Varianza entre el Consumo de Tabaco de Jóvenes Universitarios de la Universidad de Córdoba y la Valoración de Posibles Riesgos Asociados

\begin{tabular}{|c|c|c|c|c|}
\hline RIESGOS & $\begin{array}{l}\text { NIVEL DE } \\
\text { CONSUMO }\end{array}$ & $\begin{array}{l}\text { PUNTUACION } \\
\text { MEDIA (1 a 5) }\end{array}$ & $F(2,998)$ & $\mathbf{P}$ \\
\hline \multirow{3}{*}{ Accidentes de tráfico } & Nunca & 2,51 & \multirow{3}{*}{21,930} & \multirow{3}{*}{$0,000^{*}$} \\
\hline & Esporádicamente & 2,23 & & \\
\hline & Habitualmente & 1,92 & & \\
\hline \multirow{3}{*}{$\begin{array}{l}\text { Problemas psicológicos y } \\
\text { físicos graves }\end{array}$} & Nunca & 4,10 & \multirow{3}{*}{1,356} & \multirow{3}{*}{0,258} \\
\hline & Esporádicamente & 3,98 & & \\
\hline & Habitualmente & 3,99 & & \\
\hline \multirow{3}{*}{$\begin{array}{l}\text { Problemas con las relaciones } \\
\text { interpersonales }\end{array}$} & Nunca & 2,83 & \multirow{3}{*}{16,446} & \multirow{3}{*}{$0,000^{*}$} \\
\hline & Esporádicamente & 2,26 & & \\
\hline & Habitualmente & 2,39 & & \\
\hline \multirow{3}{*}{ Problemas legales } & Nunca & 2,26 & \multirow{3}{*}{8,875} & \multirow{3}{*}{$0,000^{*}$} \\
\hline & Esporádicamente & 1,88 & & \\
\hline & Habitualmente & 1,90 & & \\
\hline \multirow{3}{*}{$\begin{array}{l}\text { Disminución de las } \\
\text { capacidades }\end{array}$} & Nunca & 2,47 & \multirow{3}{*}{19,179} & \multirow{3}{*}{$0,000 *$} \\
\hline & Esporádicamente & 2,01 & & \\
\hline & Habitualmente & 1,91 & & \\
\hline \multirow{3}{*}{$\begin{array}{l}\text { Problemas graves para la } \\
\text { salud }\end{array}$} & Nunca & 3,97 & \multirow{3}{*}{0,104} & \multirow{3}{*}{0,901} \\
\hline & Esporádicamente & 3,96 & & \\
\hline & Habitualmente & 3,93 & & \\
\hline
\end{tabular}

$* \mathrm{P}<0,001$

Tabla 3. Análisis de Varianza entre el Consumo de Psicofármacos de Jóvenes Universitarios de la Universidad de Córdoba y la Valoración de Posibles Riesgos Asociados

\begin{tabular}{|c|c|c|c|c|}
\hline RIESGOS & $\begin{array}{l}\text { NIVEL DE } \\
\text { CONSUMO }\end{array}$ & $\begin{array}{l}\text { PUNTUACION } \\
\text { MEDIA (1 a 5) }\end{array}$ & $F(2,998)$ & $\mathbf{P}$ \\
\hline \multirow{3}{*}{ Accidentes de tráfico } & Nunca & 4,17 & \multirow{3}{*}{9,860} & \multirow{3}{*}{$0,000^{*}$} \\
\hline & Esporádicamente & 3,92 & & \\
\hline & Habitualmente & 2,92 & & \\
\hline \multirow{3}{*}{$\begin{array}{l}\text { Problemas psicológicos y } \\
\text { físicos graves }\end{array}$} & Nunca & 4,03 & \multirow{3}{*}{13,511} & \multirow{3}{*}{$0,000^{*}$} \\
\hline & Esporádicamente & 3,64 & & \\
\hline & Habitualmente & 2,69 & & \\
\hline \multirow{3}{*}{$\begin{array}{l}\text { Problemas con las relaciones } \\
\text { interpersonales }\end{array}$} & Nunca & 3,88 & \multirow{3}{*}{20,357} & \multirow{3}{*}{$0,000^{*}$} \\
\hline & Esporádicamente & 3,16 & & \\
\hline & Habitualmente & 2,31 & & \\
\hline \multirow{3}{*}{ Problemas legales } & Nunca & 3,32 & \multirow{3}{*}{15,295} & \multirow{3}{*}{$0,000^{*}$} \\
\hline & Esporádicamente & 2,62 & & \\
\hline & Habitualmente & 1,54 & & \\
\hline \multirow{3}{*}{$\begin{array}{l}\text { Disminución de las } \\
\text { capacidades }\end{array}$} & Nunca & 4,16 & \multirow{3}{*}{24,295} & \multirow{3}{*}{$0,000^{*}$} \\
\hline & Esporádicamente & 3,84 & & \\
\hline & Habitualmente & 2,08 & & \\
\hline \multirow{3}{*}{$\begin{array}{l}\text { Problemas graves para la } \\
\text { salud }\end{array}$} & Nunca & 4,02 & \multirow{3}{*}{11,592} & \multirow{3}{*}{$0,000^{*}$} \\
\hline & Esporádicamente & 3,53 & & \\
\hline & Habitualmente & 2,92 & & \\
\hline
\end{tabular}

$* \mathrm{P}<0,001$ 
Para concretar qué riesgos concretos son los que pueden estar interviniendo en el consumo de sustancias, se ha realizado una regresión logística binaria por pasos hacia adelante para cada variable dependiente (consumo de cada una de las sustancias), agrupándolas en dos categorías (no consume y consume) y relacionándolas con las puntuaciones de los diferentes riesgos (tráfico, salud, legales, relaciones interpersonales, capacidad y riegos de salud irreversibles) (ver tabla 4).

Para el consumo de alcohol, se dicotomizó la variable en dos categorías: una, consume habitualmente, y otra, consume esporádicamente o nunca. El modelo creado para la percepción de riesgos en consumidores habituales de alcohol permite una estimación correcta del 59,4\% de los casos, $\mathrm{X}^{2}$ de 50,075 $[\mathrm{gl}(2)$, $.000]$, entrando a formar parte de la ecuación el riesgo de tener problemas en las relaciones interpersonales y el riesgo a padecer una enfermedad irreversible $\left(\mathrm{R}^{2}=\right.$ .070). La odds ratio indica que la probabilidad de que un joven universitario consumidor habitual de alcohol considere que corre algún riesgo a tener problemas en sus relaciones interpersonales $(\mathrm{OR}=.777$; IC $95 \%=.690-.875)$ o a padecer problemas de salud irreversibles $(\mathrm{OR}=.779$; IC $95 \%=.668-.909)$ es baja.

Para el consumo de tabaco, se dicotomizó la variable en dos categorías: una, consume habitualmente y esporádicamente, y otra, no consume nunca. El modelo creado para la percepción de riesgos en consumidores habituales de tabaco permite una estimación correcta del $68,1 \%$ de los casos, $\mathrm{X}^{2}$ de 59,101 [gl(3), $.000]$, entrando a formar parte de la ecuación el riesgo de tener problemas en las relaciones interpersonales, el riesgo a tener un accidente de tráfico y el riesgo a perder capacidad para realizar una tarea $\left(\mathrm{R}^{2}=.087\right)$. La odds ratio indica que la probabilidad de que un joven universitario consumidor habitual de tabaco considere que corre algún riesgo a tener un accidente de tráfico $(\mathrm{OR}=.808$; IC $95 \%=, 700-.931)$, a tener problemas en sus relaciones interpersonales $(\mathrm{OR}=$ .847 ; IC $95 \%=.745-.963)$, o perder capacidad para realizar una tarea $(\mathrm{OR}=.803$; IC $95 \%=.699-.923)$ es baja.

El modelo creado para la percepción de riesgos en consumidores habituales de psicofármacos permite una estimación correcta del 92,3\% de los casos, $\mathrm{X}^{2} \mathrm{de}$ 38,276 [gl(2), .000], entrando a formar parte de la ecuación el riesgo de tener problemas en las relaciones interpersonales y el riesgo a tener problemas legales $\left(\mathrm{R}^{2}=.099\right)$. La odds ratio indica que la probabilidad de que un joven universitario consumidor habitual de psicofármacos considere que corre algún riesgo a tener problemas en sus relaciones interpersonales $(\mathrm{OR}=.677$; IC $95 \%=.543-.843)$ y a tener problemas legales $(\mathrm{OR}=.774$; IC $95 \%=.628-.954)$ es baja.

\section{DISCUSIÓN}

Teniendo en cuenta que el objetivo de esta investigación era relacionar el consumo de sustancias legales como el alcohol, tabaco y psicofármacos con la percepción de riesgos directos e indirectos como sufrir un accidente de tráfico, padecer problemas de salud graves transitorios, problemas legales, dificultad en las relaciones interpersonales, disminución de la capacidad para realizar tareas 
y problemas de salud graves e irreversibles, se puede decir que en general aquellos jóvenes universitarios que consumen habitualmente valoran con una menor puntuación los diferentes riesgos asociados al consumo. Saber si los sujetos que consumen habitualmente tienen una menor percepción de riesgo es muy interesante y refuerza el dato empírico arrojado por otras investigaciones con muestras de edades inferiores (Espada et al., 2008; Geoffrey et al., 2008; Hernández y Solano, 2007; Martins et al., 2008; Moral et al., 2004; Moral et al., 2006; Rodríguez et al., 2008), pero conocer qué riesgos están vinculados al consumo habitual de sustancias como el tabaco, el alcohol y los psicofármacos es también de gran relevancia. Así, poder predecir qué riesgos están vinculados al consumo habitual de estas sustancias ayudará en la planificación de programas de prevención con población universitaria. Concretamente, los jóvenes universitarios consumidores habituales de alcohol no perciben como riesgos importantes tener problemas directos e indirectos derivados del consumo como problemas de salud transitorios, problemas en las relaciones interpersonales, problemas legales, disminución de la capacidad para realizar tareas y problemas de salud irreversibles, en comparación con los grupos que reconocen no consumir o hacerlo esporádicamente. Por otro lado, sí parecen coincidir a la hora de valorar como posible riesgo indirecto al consumo de alcohol, el hecho de padecer un accidente de tráfico. En este sentido, es probable que las campañas de prevención y sensibilización sobre el tema estén funcionando para que consumidores y no consumidores valoren de forma similar este riesgo. Los resultados del análisis de regresión logística identifican como riesgos asociados al consumo de alcohol problemas en las relaciones interpersonales y el riesgo a padecer una enfermedad irreversible. Esto quiere decir que aquellos jóvenes universitarios que consideran que el consumo de alcohol no afecta a sus relaciones interpersonales y/o a padecer una enfermedad grave, tendrán más probabilidades de ser consumidores habituales que aquellos que tienen una percepción alta para esos riesgos.

Los jóvenes que consumen tabaco habitual o esporádicamente no perciben como riesgo derivado del consumo tener un accidente de tráfico, tener problemas en sus relaciones interpersonales, problemas de tipo legal y problemas relacionados con la capacidad para realizar alguna tarea. Sin embargo, tanto los consumidores como los no consumidores, sí perciben y valoran con una puntuación similar problemas de salud asociados al consumo de tabaco. En este caso, los resultados de la regresión logística identifican como riesgos asociados al consumo de tabaco tener una baja percepción del riesgo para problemas con sus relaciones interpersonales, tener un accidente de tráfico y/o a perder capacidad para realizar una tarea. Es decir, que los jóvenes que valoran con una puntuación baja estos riesgos, en comparación con aquellos jóvenes que no consumen, tienen una mayor probabilidad de consumir tabaco.

Por último, los consumidores de psicofármacos valoran, en general, con una puntuación baja los posibles riesgos asociados a su consumo. Se dan diferencias significativas para todos los riesgos cuando comparamos la puntuación media que cada uno de los grupos le otorga a cada uno de los posibles riesgos. El gru- 
po que no consume es el que mayor percepción de riesgos presenta. Para esta sustancia, el análisis de regresión señala como factores de riesgo, problemas en las relaciones interpersonales y riesgo a tener problemas legales. Es decir, que los jóvenes que no perciben como riesgo que se vean deterioradas sus relaciones interpersonales o tener problemas legales, tendrán más probabilidad de ser consumidores de psicofármacos.

Como limitaciones determinadas por el propio diseño del estudio, destaca la recogida de datos en el propio aula, que si bien es el entorno más accesible, también conlleva un cierto sesgo de selección que conlleva una infraestimación en los resultados, ya que los alumnos que van a clase con regularidad son los que tienen hábitos de vida más saludables (Mas et al., 2004); y el sesgo de deseabilidad social inherente al uso de autoinformes, aunque es de suponer que no será muy alto, ya que se garantizó a los participantes su anonimato (Font-Mayolas et al., 2006).

Teniendo en cuenta los avances realizados en nuestro país en materia de prevención con sustancias legales como el tabaco (Ley 28/2005, Medidas Sanitarias), el consumo de drogas en jóvenes universitarios requiere medidas específicas de prevención que vayan orientadas sobre todo a modificar la percepción del riesgo y la aceptación social, con el objetivo último de disminuir el consumo de drogas legales como el tabaco, el alcohol y los psicofármacos entre los jóvenes.

\section{AGRADECIMIENTOS}

Este trabajo ha sido posible gracias al convenio de colaboración entre la Consejería para la Igualdad y Bienestar Social de la Junta de Andalucía y la Universidad de Córdoba en materia de drogodependencias y adicciones.

\section{REFERENCIAS}

Álvarez, E., Fraile, A.M., Secades, R., Vallejo, G. y Fernández, J.R. (2005). Percepción del riesgo del consumo de drogas en escolares de enseñanza secundaria del Principado de Asturias. Colegio Oficial de Psicológos (COP). www.princast.es/salud/

Arenas, F.J., Carmona, J., Rodríguez, A. y Tatnell, R. (2002). Patrones de consumo de drogas en la Universidad de Córdoba. Córdoba: Patronato Provincial de Servicios Sociales de Córdoba.

Ballester, R., Gil, M.D. y Guirado, M.D. (2000). Behaviours and attitudes related to alcohol consumption in adolescents between 15 and 17 years old. Annual Modification Conductual, 26, 855-895.

Becoña, E. (2000). Los adolescentes y el consumo de drogas. Papeles del Psicólogo, 77, 1-5.

Cunill, M., Planes, M. y Grass, M.E. (1998). Creencias sesgadas respecto al grado de "dureza" de algunas drogas en estudiantes universitarios. Adicciones, 10 (3), 233-238. 
Espada, J.P., Pereira, J.R. y García-Fernández, J.M. (2008). Influencia de los modelos sociales en el consumo de alcohol de los adolescentes. Psicothema, 20(4), 531-537.

Fernández, J., Álvarez, E., Secades, R., Jiménez, J.M., Canada, A., Donate, I. et al. (2003). Consumo de drogas de sintesis en estudiantes de Secundaria del Principado de Asturias (Espana). Adicciones, 15, 31-37.

Font-Mayolas, S., Grass, M.E. y Planes, M. (2006). Análisis del patrón de consumo de cannabis en estudiantes universitarios. Adicciones, 18(4), 337-344.

García de Albéniz, X.A., Guerra-Gutiérrez, F., Ortega-Martínez, R., SánchezVillegas, A. y Martínez-González, M.A. (2004). Consumo de tabaco en titulados universitarios. El proyecto SUM (Seguimiento Universidad de Navarra). Gac Sanit, 18, 108-117.

Geoffrey, H., Kristin, E. y Faith, K. (2008). Drug use and meanings of risk and pleasure. Journal of Youth Studies, 10(1), 73-96.

Gil-Flores, J. (2008). Consumo de alcohol entre estudiantes de enseñanzas secundarias. Factores de riesgo y de protección. Revista de educación, 354, 291-313.

Guillén, D., Nerín, I., Mas, A., Crucelaegui, A. (2003). Estudio de la fiabilidad de una encuesta utilizada para valorar la prevalencia, los conocimientos y las actitudes sobre el tabaquismo en estudiantes de medicina. Arch Bronconeumol, 39, 159-166.

Hernández, M. y Solano, J.C. (2007). Drogodependencias: un enfoque de género y estratificación social. Inguruak. Revista de Sociología, 44, 273-289.

Irigoyen-Coll, C. (2005). Esteroides y percepción de riesgo. Relación entre el consumo de anabólicos esteroides y la percepción del riesgo en individuos que asisten a gimnasios de la zona norte de la ciudad de Buenos Aires. Revista del Instituto de Investigación de Drogodependencias. Salud y Drogas, 5 (1).

Jiménez-Muro, A., Belmonte, A., Marqueta, A., Gargallo, P. y Nerín de la Puerta, I. (2009). Consumo de drogas en estudiantes universitarios de primer curso. Adicciones, 21(1), 21-28.

Ley 28/2005, de 26 de diciembre de medidas sanitarias frente al tabaquismo y reguladora de la venta, el suministro, el consumo y la publicidad de los productos del tabaco.

Martins, S., Store, C., Alexandre, P. y Chilcoat, H. (2008). Do adolescent ecstasy users have different attitudes towars drugs when compared to marijuana users?. Drugs and Alcohol Dependence, 94 (1-3), 63-72.

Mas, A., Nerín, I., Barrueco, M., Cordero, J. Guillén, D., Jiménez-Ruiz, C. et al. (2004). Consumo de tabaco en estudiantes de sexto curso de medicina de España. Arch Bronconeumol, 40, 403-408.

Megías, E. (2004) (Dir.). La percepción social de los problemas de drogas en España. Madrid: FAD.

Ministerio de Sanidad y Consumo (2005). Estrategia nacional sobre drogas 2000-2008. Evaluación 2003[libro electrónico].http//www.mir.es/pnd/pu- 
blica/publicaciones/

Ministerio de Sanidad y Consumo (2007). Delegación del Gobierno para el Plan Nacional sobre Drogas. Secretaría General Técnica. Observatorio Español sobre Drogas. Informe 2004: Madrid.

Montero, I. y León, O. (2007). A guide for naming research studies in Psychology. International Journal of Clinical and Health Psychology, 7(3), 847-862.

Moral, M.V. y Ovejero, A. (2005). Análisis diferencial por niveles de edad de las actitudes hacia el consumo de sustancias psicoactivas en adolescentes españoles. Interamerican Journal of Psychology, 39 (3), 325-338.

Moral, M.V., Ovejero, A. y Pastor, J. (2004). Modelado familiar y del grupo de iguales sobre la experimentación juvenil con sustancias psicoactivas. Psicothema, 81, 33-68.

Moral, M.V., Rodríguez, F.J. y Sirvent, C. (2006). Factores relacionados con las actitudes juveniles hacia el consumo de alcohol y otras sustancias psicoactivas. Psicothema, 18(1), 52-58.

Novacek, J., Raskin, R. y Hogan, R. (1991). Why do adolescent use drugs? Age, sex and user differences. Journal of Youth and Adolescence, 20, 475-492.

Navarro, J, Alonso, J.M. y Del Prado, M. (2005). Los Andaluces ante Las Drogas IX. Sevillas: Consejería para la Igualdad y Bienestar Social. Junta de Andalucía.

Parker, H., Aldridge, J., y Measham, F. (1998). Illegal Leisure: the normalisation of adolescent recreational drug use. London: Routledge.

Rodríguez, J., Fernández, A., Valdés, M., Hernández, E., Ramírez, S. y Román, A. (2008). A comparison of the peers method and traditional methodologies, and risk behaviors in studies of the prevalence of drug consumption in a population of female, Chilean students. The Spanish Journal of Psychology, 11(2), 564-572.

Trujillo A. M., Forns i Santacana M. y Pérez Gómez A. (2007). Uso de sustancias y percepción de riesgo: Estudio comparativo entre jóvenes de Bogotá y Barcelona. Adicciones. 19, 2, 179-190. 